\title{
Characterizations of Generalized Quasi-Einstein Manifolds
}

\section{Sibel SULAR and Cihan ÖZGÜR}

\begin{abstract}
We give characterizations of generalized quasi-Einstein manifolds for both even and odd dimensions.
\end{abstract}

\section{Introduction}

A Riemannian manifold $(M, g),(n \geq 2)$, is said to be an Einstein manifold if its Ricci tensor $S$ satisfies the condition $S=\frac{r}{n} g$, where $r$ denotes the scalar curvature of $M$. The notion of a quasi-Einstein manifold was introduced by M. C. Chaki and R. K. Maity in [2]. A non-flat Riemannian manifold $(M, g)$, $(n \geq 2)$, is defined to be a quasi-Einstein manifold if the condition

$$
S(X, Y)=\alpha g(X, Y)+\beta A(X) A(Y)
$$

is fulfilled on $M$, where $\alpha$ and $\beta$ are scalars of which $\beta \neq 0$ and $A$ is a non-zero 1-form such that

$$
g(X, \xi)=A(X),
$$

for every vector field $X ; \xi$ being a unit vector field. If $\beta=0$, then the manifold reduces to an Einstein manifold.

The relation (1) can be written as follows

$$
Q=\alpha I+\beta A \otimes \xi,
$$

Key Words: Einstein manifold, quasi-Einstein manifold, generalized quasi-Einstein manifold. 2010 Mathematics Subject Classification: 53C25.

Received: December, 2010.

Revised: January, 2011.

Accepted: February, 2012. 
where $Q$ is the Ricci operator and $I$ is the identity function.

Quasi-Einstein manifolds arose during the study of exact solutions of the Einstein field equations as well as during considerations of quasi-umbilical hypersurfaces. For instance, the Robertson-Walker space-times are quasiEinstein manifolds. For more information about quasi-Einstein manifolds see [7], [8] and [9].

A non-flat Riemannian manifold is called a generalized quasi-Einstein manifold (see [6]), if its Ricci tensor $S$ satisfies the condition

$$
S(X, Y)=\alpha g(X, Y)+\beta A(X) A(Y)+\gamma B(X) B(Y),
$$

where $\alpha, \beta$ and $\gamma$ are certain non-zero scalars and $A, B$ are two non-zero 1forms. The unit vector fields $\xi_{1}$ and $\xi_{2}$ corresponding to the 1-forms $A$ and $B$ are defined by

$$
g\left(X, \xi_{1}\right)=A(X), g\left(X, \xi_{2}\right)=B(X),
$$

respectively, and the vector fields $\xi_{1}$ and $\xi_{2}$ are orthogonal, i.e., $g\left(\xi_{1}, \xi_{2}\right)=0$. If $\gamma=0$, then the manifold reduces to a quasi-Einstein manifold.

The generalized quasi-Einstein condition (3) can be also written as

$$
Q=\alpha I+\beta A \otimes \xi_{1}+\gamma B \otimes \xi_{2} .
$$

In [6], U. C. De and G. C. Ghosh showed that a 2-quasi umbilical hypersurface of an Euclidean space is a generalized quasi-Einstein manifold. In [11], the present authors generalized the result of De and Ghosh and they proved that a 2-quasi umbilical hypersurface of a Riemannian space of constant curvature $\widetilde{M}^{n+1}(c)$ is a generalized quasi-Einstein manifold.

Let $M$ be an $m$-dimensional, $m \geq 3$, Riemannian manifold and $p \in M$. Denote by $K(\pi)$ or $K(u \wedge v)$ the sectional curvature of $M$ associated with a plane section $\pi \subset T_{p} M$, where $\{u, v\}$ is an orthonormal basis of $\pi$. For any $n$-dimensional subspace $L \subseteq T_{p} M, 2 \leq n \leq m$, its scalar curvature $\tau(L)$ is denoted by

$$
\tau(L)=\sum_{1 \leq i<j \leq n} K\left(e_{i} \wedge e_{j}\right),
$$

where $\left\{e_{1}, \ldots, e_{n}\right\}$ is any orthonormal basis of $L$ [4]. When $L=T_{p} M$, the scalar curvature $\tau(L)$ is just the scalar curvature $\tau(p)$ of $M$ at $p$.

The well-known characterization of 4-dimensional Einstein spaces was given by I. M. Singer and J. A. Thorpe in [12] as follows:

Theorem 1.1. A Riemannian 4-manifold $M$ is an Einstein space if and only if $K(\pi)=K\left(\pi^{\perp}\right)$ for any plane section $\pi \subseteq T_{p} M$, where $\pi^{\perp}$ denotes the orthogonal complement of $\pi$ in $T_{p} M$. 
As a generalization of the Theorem 1.1, in [4], B.Y. Chen, F. Dillen, L.Verstraelen and L.Vrancken gave the following result:

Theorem 1.2. A Riemannian $2 n$-manifold $M$ is an Einstein space if and only if $\tau(L)=\tau\left(L^{\perp}\right)$ for any $n$-plane section $L \subseteq T_{p} M$, where $L^{\perp}$ denotes the orthogonal complement of $L$ in $T_{p} M$, at $p \in M$.

On the other hand, in [10] D. Dumitru obtained the following result for odd dimensional Einstein spaces:

Theorem 1.3. A Riemannian $(2 n+1)$-manifold $M$ is an Einstein space if and only if $\tau(L)+\frac{\lambda}{2}=\tau\left(L^{\perp}\right)$ for any n-plane section $L \subseteq T_{p} M$, where $L^{\perp}$ denotes the orthogonal complement of $L$ in $T_{p} M$, at $p \in M$.

Theorem 1.2 and Theorem 1.3 were generalized by C.L. Bejan in [1] as follows:

Theorem 1.4. Let $(M, g)$ be a Riemannian $(2 n+1)$-manifold, with $n \geq 2$. Then $M$ is quasi-Einstein if and only if the Ricci operator $Q$ has an eigenvector field $\xi$ such that at any $p \in M$, there exist two real numbers $a, b$ satisfying $\tau(P)+a=\tau\left(P^{\perp}\right)$ and $\tau(N)+b=\tau\left(N^{\perp}\right)$, for any $n$-plane section $P$ and $(n+1)$-plane section $N$, both orthogonal to $\xi$ in $T_{p} M$, where $P^{\perp}$ and $N^{\perp}$ denote respectively the orthogonal complements of $P$ and $N$ in $T_{p} M$.

Theorem 1.5. Let $(M, g)$ be a Riemannian $2 n$-manifold, with $n \geq 2$. Then $M$ is quasi-Einstein if and only if the Ricci operator $Q$ has an eigenvector field $\xi$ such that at any $p \in M$, there exist two real numbers $a, b$ satisfying $\tau(P)+c=\tau\left(P^{\perp}\right)$, for any n-plane section $P$ orthogonal to $\xi$ in $T_{p} M$, where $P^{\perp}$ denotes the orthogonal complement of $P$ in $T_{p} M$.

Motivated by the above studies, as generalizations of quasi-Einstein manifolds, we give characterizations of generalized quasi-Einstein manifolds for both even and odd dimensions.

\section{Characterizations of Generalized Quasi-Einstein Man- ifolds}

Now, we consider two results which characterize generalized quasi-Einstein spaces in even and odd dimensions, by generalizing the characterizations of quasi-Einstein spaces given in [1] :

Theorem 2.1. Let $(M, g)$ be a Riemannian $(2 n+1)$-manifold, with $n \geq 2$. Then $M$ is generalized quasi-Einstein if and only if the Ricci operator $Q$ has 
eigenvector fields $\xi_{1}$ and $\xi_{2}$ such that at any $p \in M$, there exist three real numbers $a, b$ and $c$ satisfying

$$
\begin{gathered}
\tau(P)+a=\tau\left(P^{\perp}\right) ; \quad \xi_{1}, \xi_{2} \in T_{p} P^{\perp} \\
\tau(N)+b=\tau\left(N^{\perp}\right) ; \quad \xi_{1} \in T_{p} N, \xi_{2} \in T_{p} N^{\perp}
\end{gathered}
$$

and

$$
\tau(R)+c=\tau\left(R^{\perp}\right) ; \quad \xi_{1} \in T_{p} R, \xi_{2} \in T_{p} R^{\perp}
$$

for any $n$-plane sections $P, N$ and $(n+1)$-plane section $R$, where $P^{\perp}, N^{\perp}$ and $R^{\perp}$ denote the orthogonal complements of $P, N$ and $R$ in $T_{p} M$, respectively, and $a=\frac{(\alpha+\beta+\gamma)}{2}, b=\frac{(\alpha-\beta+\gamma)}{2}, c=\frac{(\gamma-\alpha-\beta)}{2}$.

Proof. Assume that $M$ is a $(2 n+1)$-dimensional generalized quasi-Einstein manifold, such that

$$
S(X, Y)=\alpha g(X, Y)+\beta A(X) A(Y)+\gamma B(X) B(Y),
$$

for any vector fields $X, Y$ holds on $M$, where $A$ and $B$ are defined by

$$
g\left(X, \xi_{1}\right)=A(X), g\left(X, \xi_{2}\right)=B(X) .
$$

The equation (5) shows that $\xi_{1}$ and $\xi_{2}$ are eigenvector fields of $Q$.

Let $P \subseteq T_{p} M$ be an $n$-plane orthogonal to $\xi_{1}$ and $\xi_{2}$ and let $\left\{e_{1}, \ldots, e_{n}\right\}$ be an orthonormal basis of it. Since $\xi_{1}$ and $\xi_{2}$ are orthogonal to $P$, we can take an orthonormal basis $\left\{e_{n+1}, \ldots, e_{2 n}, e_{2 n+1}\right\}$ of $P^{\perp}$ such that $e_{2 n}=\xi_{1}$ and $e_{2 n+1}=$ $\xi_{2}$, respectively. Thus, $\left\{e_{1}, \ldots, e_{n}, e_{n+1}, \ldots, e_{2 n}, e_{2 n+1}\right\}$ is an orthonormal basis of $T_{p} M$. Then taking $X=Y=e_{i}$ in (5), we can write

$$
S\left(e_{i}, e_{i}\right)=\sum_{j=1}^{2 n+1} R\left(e_{j}, e_{i}, e_{i}, e_{j}\right)=\left\{\begin{array}{c}
\alpha, \quad 1 \leq i \leq 2 n-1 \\
\alpha+\beta, i=2 n \\
\alpha+\gamma, \quad i=2 n+1
\end{array}\right\} .
$$

By the use of (5) for any $1 \leq i \leq 2 n+1$, we can write

$$
\begin{aligned}
& S\left(e_{1}, e_{1}\right)=K\left(e_{1} \wedge e_{2}\right)+K\left(e_{1} \wedge e_{3}\right)+\ldots+K\left(e_{1} \wedge e_{2 n-1}\right)+K\left(e_{1} \wedge \xi_{1}\right)+K\left(e_{1} \wedge \xi_{2}\right)=\alpha, \\
& S\left(e_{2}, e_{2}\right)=K\left(e_{2} \wedge e_{1}\right)+K\left(e_{2} \wedge e_{3}\right)+\ldots+K\left(e_{2} \wedge e_{2 n-1}\right)+K\left(e_{2} \wedge \xi_{1}\right)+K\left(e_{2} \wedge \xi_{2}\right)=\alpha, \\
& S\left(e_{2 n-1}, e_{2 n-1}\right)=K\left(e_{2 n-1} \wedge e_{1}\right)+K\left(e_{2 n-1} \wedge e_{2}\right)+\ldots+K\left(e_{2 n-1} \wedge \xi_{1}\right)+K\left(e_{2 n-1} \wedge \xi_{2}\right)=\alpha, \\
& S\left(\xi_{1}, \xi_{1}\right)=K\left(\xi_{1} \wedge e_{1}\right)+K\left(\xi_{1} \wedge e_{2}\right)+\ldots+K\left(\xi_{1} \wedge e_{2 n-1}\right)+K\left(\xi_{1} \wedge \xi_{2}\right)=\alpha+\beta, \\
& S\left(\xi_{2}, \xi_{2}\right)=K\left(\xi_{2} \wedge e_{1}\right)+K\left(\xi_{2} \wedge e_{2}\right)+\ldots+K\left(\xi_{2} \wedge e_{2 n-1}\right)+K\left(\xi_{2} \wedge \xi_{1}\right)=\alpha+\gamma .
\end{aligned}
$$


Now, by summing up the first $n$-equations, we get

$$
2 \tau(P)+\sum_{1 \leq i \leq n<j \leq 2 n+1} K\left(e_{i} \wedge e_{j}\right)=n \alpha .
$$

By summing up the last $(n+1)$-equations, we also get

$$
2 \tau\left(P^{\perp}\right)+\sum_{1 \leq j \leq n+1<i \leq 2 n+1} K\left(e_{i} \wedge e_{j}\right)=(n+1) \alpha+\beta+\gamma .
$$

Then, by substracting the equation (6) from (7), we obtain

$$
\tau\left(P^{\perp}\right)-\tau(P)=\frac{(\alpha+\beta+\gamma)}{2} .
$$

Similarly, let $N \subseteq T_{p} M$ be an $n$-plane orthogonal to $\xi_{2}$ and let $\left\{e_{1}, \ldots, e_{n-1}, e_{n}\right\}$ be an orthonormal basis of it. Since $\xi_{2}$ is orthogonal to $N$, we can take an orthonormal basis $\left\{e_{n+1}, \ldots, e_{2 n}, e_{2 n+1}\right\}$ of $N^{\perp}$ orthogonal to $\xi_{1}$, such that $e_{n}=\xi_{1}$ and $e_{2 n+1}=\xi_{2}$, respectively. Thus, $\left\{e_{1}, \ldots, e_{n}, e_{n+1}, \ldots, e_{2 n}, e_{2 n+1}\right\}$ is an orthonormal basis of $T_{p} M$. By making use of the above $(2 n+1)$ equations for $S\left(e_{i}, e_{i}\right), 1 \leq i \leq 2 n+1$, from the sum of the first $n$-equations we obtain

$$
2 \tau(N)+\sum_{1 \leq i \leq n<j \leq 2 n+1} K\left(e_{i} \wedge e_{j}\right)=n \alpha+\beta
$$

and from the sum of the last $(n+1)$-equations, we have

$$
2 \tau\left(N^{\perp}\right)+\sum_{1 \leq j \leq n+1<i \leq 2 n+1} K\left(e_{i} \wedge e_{j}\right)=(n+1) \alpha+\gamma .
$$

By substracting the equation (9) from (10), we find

$$
\tau\left(N^{\perp}\right)-\tau(N)=\frac{(\alpha-\beta+\gamma)}{2} .
$$

Analogously, let $R \subseteq T_{p} M$ be an $(n+1)$-plane orthogonal to $\xi_{2}$ and let $\left\{e_{1}, \ldots, e_{n}, e_{n+1}\right\}$ be an orthonormal basis of it. Since $\xi_{2}$ is orthogonal to $R$, we can take an orthonormal basis $\left\{e_{n+2}, \ldots, e_{2 n}, e_{2 n+1}\right\}$ of $R^{\perp}$ orthogonal to $\xi_{1}$, such that $e_{n+1}=\xi_{1}$ and $e_{2 n+1}=\xi_{2}$, respectively. Thus, $\left\{e_{1}, \ldots, e_{n}, e_{n+1}, \ldots, e_{2 n}, e_{2 n+1}\right\}$ is an orthonormal basis of $T_{p} M$. Similarly writing again the above $(2 n+1)$ equations for $S\left(e_{i}, e_{i}\right), 1 \leq i \leq 2 n+1$, from the sum of the first $(n+1)$ equations we get

$$
2 \tau(R)+\sum_{1 \leq i \leq n+1<j \leq 2 n+1} K\left(e_{i} \wedge e_{j}\right)=(n+1) \alpha+\beta
$$


and from the sum of the last $n$-equations, we have

$$
2 \tau\left(R^{\perp}\right)+\sum_{1 \leq j \leq n<i \leq 2 n+1} K\left(e_{i} \wedge e_{j}\right)=n \alpha+\gamma
$$

Again by substracting (11) from (12), it follows that

$$
\tau\left(R^{\perp}\right)-\tau(R)=\frac{(\gamma-\alpha-\beta)}{2} .
$$

Therefore the direct statement is satisfied for

$$
a=\frac{(\alpha+\beta+\gamma)}{2}, \quad b=\frac{(\alpha-\beta+\gamma)}{2} \quad \text { and } \quad c=\frac{(\gamma-\alpha-\beta)}{2} .
$$

Conversely, let $v$ be an arbitrary unit vector of $T_{p} M$, at $p \in M$, orthogonal to $\xi_{1}$ and $\xi_{2}$. We take an orthonormal basis $\left\{e_{1}, \ldots, e_{n}, e_{n+1}, \ldots, e_{2 n}, e_{2 n+1}\right\}$ of $T_{p} M$ such that $v=e_{1}, e_{n+1}=\xi_{1}$ and $e_{2 n+1}=\xi_{2}$. We consider $n$-plane section $N$ and $(n+1)$-plane section $R$ in $T_{p} M$ as follows

$$
N=\operatorname{span}\left\{e_{2}, \ldots, e_{n+1}\right\}
$$

and

$$
R=\operatorname{span}\left\{e_{1}, \ldots, e_{n+1}\right\},
$$

respectively. Then we have

$$
N^{\perp}=\operatorname{span}\left\{e_{1}, e_{n+2}, \ldots, e_{2 n}, e_{2 n+1}\right\}
$$

and

$$
R^{\perp}=\operatorname{span}\left\{e_{n+2}, \ldots, e_{2 n}, e_{2 n+1}\right\} .
$$

After some calculations we get

$$
\begin{aligned}
S(v, v)= & {\left[K\left(e_{1} \wedge e_{2}\right)+K\left(e_{1} \wedge e_{3}\right)+\ldots+K\left(e_{1} \wedge e_{n+1}\right)\right] } \\
& +\left[K\left(e_{1} \wedge e_{n+2}\right)+\ldots+K\left(e_{1} \wedge e_{2 n}\right)+K\left(e_{1} \wedge e_{2 n+1}\right)\right] \\
= & {\left[\tau(R)-\sum_{2 \leq i<j \leq n+1} K\left(e_{i} \wedge e_{j}\right)\right]+\left[\tau\left(N^{\perp}\right)-\sum_{n+2 \leq i<j \leq 2 n+1} K\left(e_{i} \wedge e_{j}\right)\right] } \\
= & {\left[\tau\left(R^{\perp}\right)-c-\tau(N)\right]+\left[\tau(N)+b-\tau\left(R^{\perp}\right)\right]=b-c . }
\end{aligned}
$$

Therefore $S(v, v)=b-c$, for any unit vector $v \in T_{p} M$, ortohogonal to $\xi_{1}$ and $\xi_{2}$. Then we can write for any $1 \leq i \leq 2 n+1$,

$$
S\left(e_{i}, e_{i}\right)=b-c
$$


Since $S(v, v)=(b-c) g(v, v)$ for any unit vector $v \in T_{p} M$ orthogonal to $\xi_{1}$ and $\xi_{2}$, it follows that

$$
S(X, X)=(b-c) g(X, X)+(a-b) A(X) A(X)
$$

and

$$
S(Y, Y)=(b-c) g(Y, Y)+(a+c) B(Y) B(Y),
$$

for any $X \in\left[\operatorname{span}\left\{\xi_{1}\right\}\right]^{\perp}$ and $Y \in\left[\operatorname{span}\left\{\xi_{2}\right\}\right]^{\perp}$, where $A$ and $B$ denote dual forms of $\xi_{1}$ and $\xi_{2}$ with respect to $g$, respectively.

In view of the equations (13) and (14), we get from their symmetry that $S$ with tensors $(b-c) g+(a-b) A \otimes A$ and $(b-c) g+(a+c) B \otimes B$ must coincide on the complement of $\xi_{1}$ and $\xi_{2}$, respectively, that is,

$$
S(X, Y)=(b-c) g(X, Y)+(a-b) A(X) A(Y)+(a+c) B(X) B(Y),
$$

for any $X, Y \in\left[\operatorname{span}\left\{\xi_{1}, \xi_{2}\right\}\right]^{\perp}$.

Since $\xi_{1}$ and $\xi_{2}$ are eigenvector fields of $Q$, we also have

$$
S\left(X, \xi_{1}\right)=0
$$

and

$$
S\left(Y, \xi_{2}\right)=0,
$$

for any $X, Y \in T_{p} M$ orthogonal to $\xi_{1}$ and $\xi_{2}$. Thus, we can extend the equation (15) to

$$
S(X, Z)=(b-c) g(X, Z)+(a-b) A(X) A(Z)+(a+c) B(X) B(Z),
$$

for any $X \in\left[\operatorname{span}\left\{\xi_{1}, \xi_{2}\right\}\right]^{\perp}$ and $Z \in T_{p} M$.

Now, let consider the $n$-plane section $P$ and $(n+1)$-plane section $R$ in $T_{p} M$ as follows

$$
P=\operatorname{span}\left\{e_{1}, \ldots, e_{n}\right\}
$$

and

$$
R=\operatorname{span}\left\{e_{1}, \ldots, e_{n}, \xi_{1}\right\},
$$

respectively. Then we have

$$
P^{\perp}=\operatorname{span}\left\{\xi_{1}, e_{n+2}, \ldots, e_{2 n+1}\right\}
$$

and

$$
R^{\perp}=\operatorname{span}\left\{e_{n+2}, \ldots, e_{2 n}, e_{2 n+1}\right\} .
$$


Similarly after some calculations we obtain

$$
\begin{aligned}
S\left(\xi_{1}, \xi_{1}\right)= & {\left[K\left(\xi_{1} \wedge e_{1}\right)+K\left(\xi_{1} \wedge e_{2}\right)+\ldots+K\left(\xi_{1} \wedge e_{n}\right)\right] } \\
& +\left[K\left(\xi_{1} \wedge e_{n+2}\right)+\ldots+K\left(\xi_{1} \wedge e_{2 n}\right)+K\left(\xi_{1} \wedge e_{2 n+1}\right)\right] \\
= & {\left[\tau(R)-\sum_{1 \leq i<j \leq n} K\left(e_{i} \wedge e_{j}\right)\right]+\left[\tau\left(P^{\perp}\right)-\sum_{n+2 \leq i<j \leq 2 n+1} K\left(e_{i} \wedge e_{j}\right)\right] } \\
= & {\left[\tau\left(R^{\perp}\right)-c-\tau(P)\right]+\left[\tau(P)+a-\tau\left(R^{\perp}\right)\right]=a-c . }
\end{aligned}
$$

Then, we can write

$$
S\left(\xi_{1}, \xi_{1}\right)=(b-c) g\left(\xi_{1}, \xi_{1}\right)+(a-b) A\left(\xi_{1}\right) A\left(\xi_{1}\right) .
$$

Analogously, let consider $n$-plane sections $P$ and $N$ in $T_{p} M$ as follows

$$
P=\operatorname{span}\left\{e_{1}, \ldots, e_{n}\right\}
$$

and

$$
N=\operatorname{span}\left\{e_{n+1}, \ldots, e_{2 n}\right\},
$$

respectively. Therefore we have

$$
P^{\perp}=\operatorname{span}\left\{e_{n+1}, \ldots, e_{2 n}, \xi_{2}\right\}
$$

and

$$
N^{\perp}=\operatorname{span}\left\{e_{1}, \ldots, e_{n}, \xi_{2}\right\} .
$$

Similarly after some calculations we get

$$
\begin{aligned}
S\left(\xi_{2}, \xi_{2}\right)= & {\left[K\left(\xi_{2} \wedge e_{1}\right)+K\left(\xi_{2} \wedge e_{2}\right)+\ldots+K\left(\xi_{2} \wedge e_{n}\right)\right] } \\
& +\left[K\left(\xi_{2} \wedge e_{n+1}\right)+\ldots+K\left(\xi_{2} \wedge e_{2 n}\right)\right] \\
= & {\left[\tau\left(N^{\perp}\right)-\sum_{1 \leq i<j \leq n} K\left(e_{i} \wedge e_{j}\right)\right]+\left[\tau\left(P^{\perp}\right)-\sum_{n+1 \leq i<j \leq 2 n} K\left(e_{i} \wedge e_{j}\right)\right] } \\
= & {[\tau(N)+b-\tau(P)]+[\tau(P)+a-\tau(N)]=a+b . }
\end{aligned}
$$

Then we may write

$$
S\left(\xi_{2}, \xi_{2}\right)=(b-c) g\left(\xi_{2}, \xi_{2}\right)+(a+c) B\left(\xi_{2}\right) B\left(\xi_{2}\right) .
$$

By making use of the equations (16), (17) and (18), we obtain from the symmetry of the Ricci tensor $S$

$$
S(X, Y)=(b-c) g(X, Y)+(a-b) A(X) A(Y)+(a+c) B(X) B(Y),
$$

for any $X, Y \in T_{p} M$. Thus, $M$ is a generalized quasi-Einstein manifold for $\alpha=b-c, \beta=a-b$ and $\gamma=a+c$, which finishes the proof of the theorem. 
Similar to the proof of Theorem 2.1, we can give the following theorem for an even dimensional generalized quasi-Einstein manifold:

Theorem 2.2. Let $(M, g)$ be a Riemannian $2 n$-manifold, with $n \geq 2$. Then $M$ is generalized quasi-Einstein if and only if the Ricci operator $Q$ has eigenvector fields $\xi_{1}$ and $\xi_{2}$ such that at any $p \in M$, there exist three real numbers $a, b$ and c satisfying

$$
\begin{gathered}
\tau(P)+a=\tau\left(P^{\perp}\right) ; \quad \xi_{1}, \xi_{2} \in T_{p} P^{\perp} \\
\tau(N)+b=\tau\left(N^{\perp}\right) ; \quad \xi_{1}, \xi_{2} \in T_{p} N^{\perp}
\end{gathered}
$$

and

$$
\tau(R)+c=\tau\left(R^{\perp}\right) ; \quad \xi_{1} \in T_{p} R, \xi_{2} \in T_{p} R^{\perp}
$$

for any $n$-plane sections $P, R$ and $(n-1)$-plane section $N$, where $P^{\perp}, N^{\perp}$ and $R^{\perp}$ denote the orthogonal complements of $P, N$ and $R$ in $T_{p} M$, respectively and $a=\frac{(\beta+\gamma)}{2}, b=\frac{(2 \alpha+\beta+\gamma)}{2}, c=\frac{(\gamma-\beta)}{2}$.

Proof. Let $P$ and $R$ be $n$-plane sections and $N$ be an $(n-1)$-plane section such that

$$
\begin{gathered}
P=\operatorname{span}\left\{e_{1}, \ldots, e_{n}\right\} \\
R=\operatorname{span}\left\{e_{n+1}, \ldots, e_{2 n}\right\},
\end{gathered}
$$

and

$$
N=\operatorname{span}\left\{e_{2}, \ldots, e_{n}\right\},
$$

respectively. Therefore the orthogonal complements of these sections can be written as

$$
\begin{gathered}
P^{\perp}=\operatorname{span}\left\{e_{n+1}, \ldots, e_{2 n}\right\} \\
R^{\perp}=\operatorname{span}\left\{e_{1}, \ldots, e_{n}\right\},
\end{gathered}
$$

and

$$
N^{\perp}=\operatorname{span}\left\{e_{1}, e_{n+1} \ldots, e_{2 n}\right\} .
$$

Then the proof is similar to the proof of Theorem 2.1 .

\section{References}

[1] C. L. Bejan, Characterizations of quasi-Einstein manifolds, An. Ştiinţ. Univ. Al. I. Cuza Iaşi. Mat. (N.S.) 53(2007), suppl. 1, 67-72.

[2] M. C. Chaki and R. K. Maity, On quasi Einstein manifolds, Publ. Math. Debrecen 57(2000), no. 3-4, 297-306. 
[3] B. Y. Chen, F. Dillen, L. Verstraelen and L. Vrancken, Characterizations of Riemannian space forms, Einstein spaces and conformally flat spaces, Proc. Am. Math. Soc., 128(1999), no. 2, 589-598.

[4] B. Y. Chen, Some new obstructions to minimal and Lagrangian, isometric immersions, Japan J. Math. 26(2000), 105-127.

[5] U. C. De and G. C. Ghosh, On quasi Einstein manifolds, Period. Math. Hungar. 48(2004), no. 1-2, 223-231.

[6] U. C. De and G. C. Ghosh, On generalized quasi Einstein manifolds, Kyungpook Math. J. 44(2004), no. 4, 607-615.

[7] U. C. De and G. C. Ghosh, Some global properties of generalized quasi-Einstein manifolds, Ganita 56(2005), no. 1, 65-70.

[8] G. C. Ghosh, U. C. De and T. Q. Binh, Certain curvature restrictions on a quasi Einstein manifold, Publ. Math. Debrecen 69(2006), no. 1-2, 209-217.

[9] U. C. De, J. Sengupta and D. Saha, Conformally flat quasi-Einstein spaces, Kyungpook Math. J. 46(2004), no. 3, 417-423.

[10] D. Dumitru, On Einstein spaces of odd dimension, Bul. Univ. Transilvania Brasov, 14(49)(2007), 95-97.

[11] C. Özgür and S. Sular, On some properties of generalized quasi-Einstein manifolds, Indian J. Math. 50 (2008), no. 2, 297-302.

[12] I. M. Singer and J. A. Thorpe, The curvature of 4-dimensional Einstein spaces, Global Analysis, Princeton University Press (1969), 355-365.

Sibel SULAR,

Department of Mathematics,

Balkesir University,

10145, Balkesir, Turkey.

Email: csibel@balikesir.edu.tr

Cihan ÖZGÜR,

Department of Mathematics,

Balkesir University,

10145, Balkesir, Turkey.

Email: cozgur@balikesir.edu.tr 